\title{
Contributions du sommeil à la consolidation mnésique
}

\author{
Pierre Maquet, Luca Matarazzo, Ariane Foret, Laura Mascetti, Anahita Shaffii-Le Bourdiec et Vincenzo Muto \\ Centre de Recherches du Cyclotron, Université de Liège, Bâtiment 30 - Sart Tilman 4000 Liège 1, Belgique
}

Auteur correspondant: Pierre Maquet, pmaquet@ulg.ac.be

Reçu le 26 november 2009

\begin{abstract}
Résumé - De nombreuses données expérimentales montrent que le sommeil participe à la consolidation mnésique. La recherche tente actuellement de décrire les mécanismes cellulaires qui expliquent cet effet, en caractérisant les processus associés aux oscillations du sommeil, par exemple les ondes lentes et les fuseaux du sommeil lent. Actuellement, deux hypothèses sont à l'étude. La première suppose qu'une recalibration synaptique s'effectue durant les ondes lentes; la seconde suppose une réorganisation de la trace mnésique au niveau systémique à la faveur des fuseaux corticaux et ondes à front raide hippocampiques, impliquant un échange d'information entre l'hippocampe et le néocortex.
\end{abstract}

Mots clés : Éveil / sommeil / mémoire / consolidation

Abstract - Contribution of sleep to learning and memory.

A growing body of evidence indicates that sleep promotes memory consolidation. Although the first experimental evidence for this positive influence of sleep on memory was collected more than a century ago, the potential underlying neural mechanisms begin only to be conceptualized and experimentally characterized. A first hypothesis contrasted the influence of non rapid eye movement (NREM) sleep and rapid eye movement (REM) sleep on declarative and procedural memories, respectively. As the understanding of the effects of sleep on memory consolidation during sleep progressed, the hypotheses were increasingly framed in terms of neural processes occurring with NREM and REM sleep, especially associated with phasic events such as slow waves, spindles or phasic REM sleep. This paper reviews two of these hypothesis : the synaptic downscaling and the systemic consolidation during non REM sleep.

Key words: Wakefulness / sleep / memory / consolidation

\section{Introduction}

2 L'éveil et le sommeil sont encore souvent perçus comme des états de fonctionnement cérébral indépendants et antithétiques, le premier associé au jour, à la conscience et à l'action, le second évoquant la nuit, la perte de conscience, une réduction de l'activité cérébrale et une vie végétative dévolue au repos et à la restauration tissulaire.

Cette vision est erronée à double titre. Premièrement, durant le sommeil, l'activité cérébrale reste considérable. Durant le sommeil lent, elle est rythmée par une oscillation lente $(<1 \mathrm{~Hz})$ qui, chez l'animal, se caractérise par l'alternance de périodes de dépolarisation (états " $u p ")$ et d'hyperpolarisation (états "down") survenant de manière synchrone dans de larges populations corticales (Steriade et al., 1993a, 1993b, 1993c). Les décharges neuronales toniques associées aux phases de dépolarisation, séparées par le silence neuronal des périodes d'hyperpolarisation, sont à l'origine de l'enregistrement en électroencéphalographie des ondes lentes de grande amplitude caractéristiques de cette phase de sommeil. L'oscillation lente organise la production d'autres rythmes tels les fuseaux du sommeil (Steriade \& Amzica, 1998) et les ondes à front raide hippocampiques (Isomura et al., 2006), qui surviennent plus volontiers durant les phases actives. Chez l'Homme, 
bien qu'en moyenne la consommation énergétique cérébrale soit moins élevée en sommeil lent qu'à l'éveil (Maquet et al., 1990), les enregistrements intracrâniens (Cash et al., 2009) et des études de neuro-imagerie (Dang-Vu et al., 2008) ont permis de confirmer la présence d'une activité phasique, synchrone aux ondes lentes, durant le sommeil lent. De la même manière, les régions cérébrales qui s'activent de manière synchrone aux fuseaux ont été récemment caractérisées par résonance magnétique fonctionnelle (Schabus et al., 2007). Quant au sommeil paradoxal, il est associé chez l'animal à une activité neuronale tonique très similaire à l'activité d'éveil (Steriade \& McCarley, 2005) et les besoins métaboliques du cerveau en sommeil paradoxal sont équivalents à ceux de l'éveil (Maquet et al., 1990).

Deuxièmement, l'éveil et le sommeil interagissent de manière intime. L'influence de la qualité du sommeil sur les performances diurnes est bien connue. En revanche, l'influence de l'expérience acquise à la veille sur la qualité du sommeil subséquent a été reconnue plus récemment. Les travaux menés durant la dernière décade suggèrent que les modifications d'activité cérébrale observées pendant le sommeil après un apprentissage participent à la consolidation des traces mnésique fraîches. Le présent article tente de résumer les données et hypothèses récentes à ce sujet.

\section{Effets comportementaux}

Il est généralement admis que la récupération d'une information apprise est meilleure si l'individu a dormi après l'apprentissage, plutôt que de rester éveillé. Cet avantage mnésique lié au sommeil a été rapporté pour des tâches de mémoire déclarative (paires de mots) (Gais \& Born, 2004b), de mémoire émotionnelle (Wagner et al., 2001, 2006), de mémoire procédurale visuo-motrice (apprentissage d'une séquence motrice) (Walker et al., 2002), d'adaptation visuo-motrice (Huber et al., 2004), et d'apprentissage perceptuel visuel (Karni et al., 1994; Gais et al., 2000; Stickgold et al., 2000). Il existe cependant quelques tâches dont la performance ne semble pas spécifiquement améliorée par le sommeil (Gottselig et al., 2004).

Les observations initiales suggéraient que la mémoire déclarative était particulièrement sensible aux effets du sommeil lent tandis que la mémoire procédurale bénéficiait particulièrement du sommeil paradoxal (Plihal \& Born, 1999). Des observations ultérieures ont infirmé cette hypothèse en montrant, par exemple, que des habiletés motrices s'amélioraient en proportion de la profondeur du sommeil lent obtenu après l'entraînement (Huber et al., 2004).

Les hypothèses actuelles ne s'attachent plus à attribuer le traitement des traces mnésiques à certains

stades de sommeil mais plutôt à caractériser l'effet sur la consolidation mnésique de l'activité neuronale liée à des oscillations spécifiques au sommeil. Ainsi, l'avantage mnésique lié au sommeil est-il maintenant attribué aux activités neuronales associées aux ondes lentes (Huber et al., 2004) et aux fuseaux du sommeil lent (Gais et al., 2002; Schabus et al., 2004; Fogel \& Smith, 2006), aux ondes pontines du sommeil paradoxal (Datta, 2000) ou aux contextes de neuromodulation particuliers aux phases de sommeil (Gais \& Born, 2004a).

\section{Mécanismes neuronaux de la consolidation mnésique liée au sommeil}

La majorité des travaux récents a tenté de mieux comprendre l'effet des oscillations du sommeil lent (ondes lentes et fuseaux) sur la consolidation mnésique. Deux hypothèses sont en général avancées, celle de la recalibration synaptique et celle de la consolidation systémique.

\section{Recalibration synaptique}

Cette hypothèse (Tononi \& Cirelli, 2003, 2006) postule que les périodes d'éveil s'accompagnent en moyenne d'une potentiation synaptique dans la grande majorité des circuits cérébraux. Cette augmentation accrue de transmission synaptique, principalement glutamatergique, ne serait pas tenable à long terme en raison du coût énergétique qu'elle implique. Durant le sommeil, l'oscillation lente $(<1 \mathrm{~Hz})$ serait associée à une recalibration globale des poids synaptiques à des niveaux compatibles avec un fonctionnement neuronal pérenne. Cette recalibration, en préservant les différences d'efficacité entre synapses, participerait ainsi à la consolidation mnésique.

En accord avec cette hypothèse, il a été montré que la puissance des ondes lentes est augmentée pendant le sommeil lent subséquent à un apprentissage visuo-moteur, spécifiquement dans la région pariétale supposée responsable de l'apprentissage et suspectée d'avoir subi une potentiation synaptique pendant l'entraînement (Huber et al., 2004). À l'inverse, l'immobilisation d'un membre durant la journée s'accompagne d'une diminution de l'activité des ondes lentes durant le sommeil subséquent, principalement dans la région sensorimotrice controlatérale (Huber et al., 2006).

L'hypothèse a récemment reçu confirmation de recherches menées chez le rongeur. La pente et l'amplitude de la réponse évoquée par la stimulation corticale, indicateurs neurophysiologiques de la puissance
65

66

67 
1 synaptique, sont accrus à l'éveil et diminuent en proportion de la quantité d'ondes lentes générées pendant le sommeil (Vyazovskiy et al., 2008). De la même manière, les marqueurs moléculaires de la transmission synaptique AMPA (GluR1) et de la potentiation synaptique glutamatergique (phosphorylation des récepteurs AMPA, de la CAM kinase II) sont présents à un niveau plus élevé après une période de veille qu'après une phase de sommeil (Vyazovskiy et al., 2008). Des modifications similaires ont été observées chez la Drosophile (Gilestro et al., 2009).

\section{Consolidation systémique}

L'hypothèse classique de la consolidation des traces mnésiques hippocampo-dépendantes postule que la trace mnésique est progressivement réorganisée au cours du temps. (Frankland \& Bontempi, 2005). Le souvenir est initialement encodé dans des réseaux hippocampo-néocorticaux. Des réactivations successives de ces réseaux renforcent graduellement les connections cortico-corticales, de telle manière qu'à long terme, le souvenir est encodé dans des circuits purement corticaux. Ces réactivations spontanées des réseaux hippocampo-corticaux surviendraient préférentiellement durant le sommeil (Maquet, 2001).

Effectivement, chez le rat, des séquences de décharges neuronales enregistrées durant le sommeil dans l'hippocampe (Hirase et al., 2001) et dans le cortex (Euston \& McNaughton, 2006) reproduisent les séquences observées préalablement pendant l'exploration d'un labyrinthe. Ces répétitions de séquences sont coordonnées entre l'hippocampe et le cortex, occipital (Ji \& Wilson, 2007), frontal (Peyrache et al., 2009) ainsi que le striatum (Lansink et al., 2009). Elles surviennent de manière préférentielle durant les ondes à front raide hippocampiques, associées à des « ripples» (trains d'ondes à haute fréquence). Aussi, après l'exploration d'un labyrinthe, la suppression des ripples durant le sommeil détériore-t-elle les capacités ultérieures de navigation (Girardeau et al., 2009).

Chez l'homme, des études de neuro-imagerie ont montré qu'après l'exploration d'un labyrinthe virtuel, l'activité de l'hippocampe était accrue pendant le sommeil lent en proportion du gain de performance de navigation observé entre l'entraînement initial et le retest le lendemain (Peigneux et al., 2004). Ces « réactivations» ont également été observées durant le sommeil paradoxal subséquent à un apprentissage de séquences motrices, dans des aires corticales (occipitales, prémotrices) qui étaient recrutées à l'entraînement (Maquet et al., 2000).

En outre, la privation de sommeil durant la nuit qui suit l'apprentissage perturbe la consolidation mnésique systémique : elle détériore la performance mnésique ultérieure et modifie les réponses cérébrales lors du rappel du souvenir (Orban et al., 2006; Gais et al., 2007; Sterpenich et al., 2007), même après des délais de plusieurs mois (Gais et al., 2007; Sterpenich et al., 2007).

\section{La consolidation ne survient pas exclusivement à l'éveil}

Ces données n'impliquent aucunement l'intervention exclusive du sommeil dans la consolidation mnésique. Il est très probable que la consolidation mnésique progresse tant à l'éveil que durant le sommeil. Des enregistrements multi-unitaires chez le macaque ont montré des réactivations de patrons de décharges dans les cortex somato-sensoriels, moteurs et pariétaux à la suite d'un apprentissage spatial (Hoffman \& McNaughton, 2002). Des résultats similaires ont été obtenus chez l'homme en imagerie par résonance magnétique fonctionnelle. Ils suggèrent que la trace mnésique se modifie, à l'éveil, dans l'heure qui suit l'entraînement à la navigation dans un labyrinthe ou un apprentissage de séquences motrices (Peigneux et al., 2006).

\section{Conclusion}

Le sommeil, loin d'être une période d'inactivité cérébrale, participe activement au traitement et à la consolidation des traces mnésiques fraiches, tant au niveau synaptique que systémique. Ces résultats résumés dans le présent article plaident pour un respect plus grand des périodes de sommeil dans une société industrielle qui exige de l'individu une performance cognitive optimale sur des périodes étendues du nycthémère.

\section{Références}

Cash S.S., Halgren E., Dehghani N., Rossetti A.O., Thesen T., Wang C., Devinsky O., Kuzniecky R., Doyle W., Madsen J.R., Bromfield E., Eross L., Halasz P., Karmos G., Csercsa R., Wittner L., Ulbert I., The human K-complex represents an isolated cortical downstate. Science, 2009, 324, 1084-1087.

Dang-Vu T.T., Schabus M., Desseilles M., Albouy G., Boly M., Darsaud A., Gais S., Rauchs G., Sterpenich V., Vandewalle G., Carrier J., Moonen G., Balteau E., Degueldre C., Luxen A., Phillips C., Maquet P., Spontaneous neural activity during human slow wave sleep. Proc Natl Acad Sci USA, 2008, 105, $15160-15165$.
77 78 79 80 81 
Datta S., Avoidance task training potentiates phasic pontine-wave density in the rat: A mechanism for sleep-dependent plasticity. J Neurosci, 2000, 20, $8607-8613$

Euston D.R., McNaughton B.L., Apparent encoding of sequential context in rat medial prefrontal cortex is accounted for by behavioral variability. J Neurosci, 2006, 26, 13143-13155.

Fogel S.M., Smith C.T., Learning-dependent changes in sleep spindles and stage 2 sleep. J Sleep Res, 2006, 15, 250-255.

Frankland P.W., Bontempi B., The organization of recent and remote memories. Nat Rev Neurosci, 2005, 6, 119-130.

Gais S., Born J., Low acetylcholine during slow-wave sleep is critical for declarative memory consolidation. Proc Natl Acad Sci USA, 2004a, 101, 2140-2144.

Gais S., Born J., Declarative memory consolidation : mechanisms acting during human sleep. Learn Mem, 2004b, 11, 679-685.

Gais S., Albouy G., Boly M., Dang-Vu T.T., Darsaud A., Desseilles M., Rauchs G., Schabus M., Sterpenich V., Vandewalle G., Maquet P., Peigneux P., Sleep transforms the cerebral trace of declarative memories. Proc Natl Acad Sci USA, 2007, 104, 18778-18783.

Gais S., Molle M., Helms K., Born J., Learning-dependent increases in sleep spindle density. J Neurosci, 2002, 22, 6830-6834.

Gais S., Plihal W., Wagner U., Born J., Early sleep triggers memory for early visual discrimination skills. Nat Neurosci, 2000, 3, 1335-1339.

Gilestro G.F., Tononi G., Cirelli C., Widespread changes in synaptic markers as a function of sleep and wakefulness in Drosophila. Science, 2009, 324, 109-112.

Girardeau G., Benchenane K., Wiener S.I., Buzsaki G., Zugaro M.B., Selective suppression of hippocampal ripples impairs spatial memory. Nat Neurosci, 2009, $12,1222-1223$.

Gottselig J.M., Hofer-Tinguely G., Borbely A.A., Regel S.J., Landolt H.P., Retey J.V., Achermann P., Sleep and rest facilitate auditory learning. Neuroscience, 2004, 127, 557-561.

Hirase H., Leinekugel X., Czurko A., Csicsvari J., Buzsaki G., Firing rates of hippocampal neurons are preserved during subsequent sleep episodes and modified by novel awake experience. Proc Natl Acad Sci USA, 2001, 98, 9386-9390.

Hoffman K.L., McNaughton B.L., Coordinated reactivation of distributed memory traces in primate neocortex. Science, 2002, 297, 2070-2073.

Huber R., Ghilardi M.F., Massimini M., Tononi G., Local sleep and learning. Nature, 2004, 430, 78-81.
Huber R., Ghilardi M.F., Massimini M., Ferrarelli F., Riedner B.A., Peterson M.J., Tononi G., Arm immobilization causes cortical plastic changes and locally decreases sleep slow wave activity. Nat Neurosci, 2006, 9, 1169-1176.

Isomura Y., Sirota A., Ozen S., Montgomery S., Mizuseki K., Henze D.A., Buzsaki G., Integration and segregation of activity in entorhinal-hippocampal subregions by neocortical slow oscillations. Neuron, 2006, $52,871-882$

Ji D., Wilson M.A., Coordinated memory replay in the visual cortex and hippocampus during sleep. Nat Neurosci, 2007, 10, 100-107.

Karni A., Tanne D., Rubenstein B.S., Askenasy J.J., Sagi D., Dependence on REM sleep of overnight improvement of a perceptual skill. Science, 1994, 265, 679-682.

Lansink C.S., Goltstein P.M., Lankelma J.V., McNaughton B.L., Pennartz C.M., Hippocampus leads ventral striatum in replay of place-reward information. PLoS Biol, 2009, 7, e1000173.

Maquet P., The role of sleep in learning and memory. Science, 2001, 294, 1048-1052.

Maquet P., Dive D., Salmon E., Sadzot B., Franco G., Poirrier R., von Frenckell R., Franck G., Cerebral glucose utilization during sleep-wake cycle in man determined by positron emission tomography and $[18 \mathrm{~F}] 2$ fluoro-2-deoxy-D-glucose method. Brain Res, 1990, $513,136-143$.

Maquet P., Laureys S., Peigneux P., Fuchs S., Petiau C., Phillips C., Aerts J., Del Fiore G., Degueldre C., Meulemans T., Luxen A., Franck G., Van Der Linden M., Smith C., Cleeremans A., Experience-dependent changes in cerebral activation during human REM sleep. Nat Neurosci, 2000, 3, 831-836.

Orban P., Rauchs G., Balteau E., Degueldre C., Luxen A., Maquet P., Peigneux P., Sleep after spatial learning promotes covert reorganization of brain activity. Proc Natl Acad Sci USA, 2006,103, 7124-7129.

Peigneux P., Laureys S., Fuchs S., Collette F., Perrin F., Reggers J., Phillips C., Degueldre C., Del Fiore G., Aerts J., Luxen A., Maquet P., Are spatial memories strengthened in the human hippocampus during slow wave sleep? Neuron, 2004, 44, 535-545.

Peigneux P., Orban P., Balteau E., Degueldre C., Luxen A., Laureys S., Maquet P., Offline persistence of memory-related cerebral activity during active wakefulness. PLoS Biol, 2006, 4, e100.

Peyrache A., Khamassi M., Benchenane K., Wiener S.I., 100 Battaglia F.P., Replay of rule-learning related neu- 101 ral patterns in the prefrontal cortex during sleep. Nat 102 Neurosci, 2009, 7, 919-926

Plihal W., Born J., Effects of early and late nocturnal sleep on priming and spatial memory. Psychophysiology, $1999,36,571-582$. 
Schabus M., Dang-Vu T.T., Albouy G., Balteau E., Boly M., Carrier J., Darsaud A., Degueldre C., Desseilles M., Gais S., Phillips C., Rauchs G., Schnakers C., Sterpenich V., Vandewalle G., Luxen A., Maquet P., Hemodynamic cerebral correlates of sleep spindles during human non-rapid eye movement sleep. Proc Natl Acad Sci USA, 2007, 104, 13164-13169.

Schabus M., Gruber G., Parapatics S., Sauter C., Klosch G., Anderer P., Klimesch W., Saletu B., Zeitlhofer J., Sleep spindles and their significance for declarative memory consolidation. Sleep, 2004, 27, 1479-1485.

Steriade M., Amzica F., Coalescence of sleep rhythms and their chronology in corticothalamic networks. Sleep Res Online, 1998, 1, 1-10.

Steriade M., McCarley R.W., Brain control of wakefulness and sleep. 2005, Kluwer Academic, New York

Steriade M., Nunez A., Amzica F., Intracellular analysis of relations between the slow $(<1 \mathrm{~Hz})$ neocortical oscillation and other sleep rhythms of the electroencephalogram. J Neurosci, 1993a, 13, 3266-3283.

Steriade M., Nunez A., Amzica F., A novel slow $(<1 \mathrm{~Hz})$ oscillation of neocortical neurons in vivo : depolarizing and hyperpolarizing components. J Neurosci, 1993b, $13,3252-3265$.

Steriade M., Contreras D., Curro Dossi R., Nunez A., The slow $(<1 \mathrm{~Hz})$ oscillation in reticular thalamic and thalamocortical neurons : scenario of sleep rhythm generation in interacting thalamic and neocortical networks. J Neurosci, 1993c, 13, 3284-3299.
Sterpenich V., Albouy G., Boly M., Vandewalle G., Darsaud A., Balteau E., Dang-Vu T.T., Desseilles M., D'Argembeau A., Gais S., Rauchs G., Schabus M., Degueldre C., Luxen A., Collette F., Maquet P., Sleeprelated hippocampo-cortical interplay during emotional memory recollection. PLoS Biol, 2007, 5, e282.

Stickgold R., James L., Hobson J.A., Visual discrimination learning requires sleep after training. Nat Neurosci, 2000, 3, 1237-1238.

Tononi G., Cirelli C., Sleep and synaptic homeostasis : a hypothesis. Brain Res Bull, 2003, 62, 143-150.

Tononi G., Cirelli C., Sleep function and synaptic homeostasis. Sleep Med Rev, 2006, 10, 49-62.

Vyazovskiy V.V., Cirelli C., Pfister-Genskow M., Faraguna U., Tononi G., Molecular and electrophysiological evidence for net synaptic potentiation in wake and depression in sleep. Nat Neurosci, 2008, 11, 200-208.

Wagner U., Gais S., Born J., Emotional memory formation is enhanced across sleep intervals with high amounts of rapid eye movement sleep. Learn Mem, 2001, 8, $112-119$.

Wagner U., Hallschmid M., Rasch B., Born J., Brief sleep after learning keeps emotional memories alive for years. Biol Psychiatry, 2006, 60, 788-790.

Walker M.P., Brakefield T., Morgan A., Hobson J.A., Stickgold R., Practice with sleep makes perfect : sleepdependent motor skill learning. Neuron, 2002, 35, 205-211. 